\title{
Young urban women and the nutrition transition in Jordan
}

\author{
Hala N Madanat ${ }^{1, *}$, Ryan Lindsay ${ }^{2}$ and Tiffany Campbell ${ }^{1}$ \\ ${ }^{1}$ Graduate School of Public Health, College of Health and Human Services, San Diego State University, 5500 \\ Campanile Drive, San Diego, CA 92182, USA: ${ }^{2}$ San Diego State University/University of California San Diego \\ Joint Doctoral Program, Public Health (Global Health), San Diego, CA, USA
}

\section{Submitted 5 February 2010: Accepted 2 June 2010: First published online 12 August 2010}

\begin{abstract}
Objective: To determine the nutrition transition stage of female Jordanian college students.

Design: A cross-sectional survey was used to assess eating styles, disordered eating attitudes and behaviours, body esteem and dissatisfaction, and media influence.

Setting: Public and private universities in Jordan.

Subjects: A total of 255 subjects were recruited through a government-initiated youth campaign.

Results: The majority of participants had a normal BMI (70.6\%) with almost all (99.4\%) reporting restrained eating behaviour. Scores on the Eating Attitudes Test (EAT-26) indicated that $45 \cdot 2 \%$ of these female college students should be screening for eating disorders. Subscales of the Body Esteem Scale (BES) showed that these women did not have substantial body esteem issues and mean scores on the Sociocultural Attitudes Towards Appearance Questionnaire (SATAQ-3) indicated that overall these women did not feel the media was dictating the way their body should look. Where Jordanian women did feel pressure from Western media, there was a $6 \cdot 7$-fold increase in the likelihood that they wanted to lose weight. In addition, $48 \cdot 2 \%$ of the female college students desired to lose weight and $14.4 \%$ desired weight gain, indicating a certain level of body dissatisfaction. Conclusions: With low levels of overweight and obesity and a propensity towards eating based on external hunger cues, college-aged Jordanian women may be less advanced in their development through the nutrition transition than the general population of women. However, high levels of restrained eating and disordered eating attitudes and behaviours indicate the need for an intervention to address healthy weight-loss strategies, assess eating disorders and help maintain healthy body esteem.
\end{abstract}

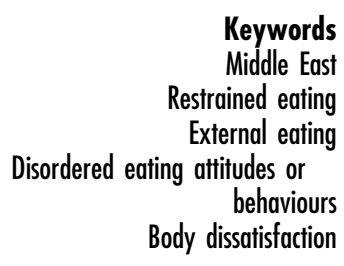

Throughout the developing world the nutrition transition is occurring at a rapid pace guided by various factors including economic changes, modernization and urbanization $^{(1-3)}$. This transition away from traditional diets that consisted largely of a healthy balance of grains, fibre, fruits and vegetables to a more Westernized diet rich in fat, sugar and salt is cause for concern for many public health officials ${ }^{(4-6)}$. Shifts in dietary patterns combined with increasingly sedentary lifestyles incurred through changes in occupation and improved technology have led to increased rates of obesity and diet-related noncommunicable diseases (such as diabetes, stroke, CVD) in countries whose previous focus had been to fight infectious disease ${ }^{(7)}$. Furthermore, Western influences encourage the transition by providing a global market that relies heavily on its ability to change attitudes and create demand with the use of media and advertising ${ }^{(8,9)}$.
Two previous studies ${ }^{(10,11)}$ have indicated that Jordan is progressing quickly through the nutrition transition, as shown by escalating rates of overweight, obesity and dietrelated non-communicable diseases. The pace of the nutrition transition has had a stronger effect on Jordanian women, who have shown higher rates of obesity than men across all age groups over the past decade ${ }^{(12)}$. In addition, Jordan's rising trend towards urbanization and a dramatically increasing younger demographic, caused by improvements in infant mortality and fertility rates, have helped to further accelerate Jordan's movement through this transition. As expected in a country going through the nutrition transition, high rates of restrained eating and disordered eating attitudes and behaviours, such as dieting and emotional eating, have been observed in the population ${ }^{(10)}$. However, the women in that sample showed 'reasonable and healthy' attitudes in regard to 
body size, preferring a 'normal' body size; and overall had normal levels of body esteem and low levels of body dissatisfaction. Researchers suggest that as Jordan moves through the nutrition transition, women will struggle between embracing more modern and Westernized cultures of diet and body size preferences that promote thinness and retaining traditional cultural norms that favour a customary diet and a normal body size ${ }^{(10)}$. Furthermore, women in Jordan are faced with an influx of media and advertising whose purpose is to influence dietary attitudes and promote Western ideals ${ }^{(8,9)}$.

Jordan's large young and urban demographic is particularly susceptible to the increasing trend of obesity and inactivity as they proceed through the transition. Studies have shown that young women's dieting behaviours and the development of eating disorders are supported by cultural values and attitudes, which become conflicted by an increasing presence of Western media and advertising and an eager acceptability to conform to modern behaviours ${ }^{(13)}$. Moreover, studies of American universities have shown higher rates of chronic dieting, binge and purging, and bulimia nervosa among female college students than in the general population ${ }^{(14)}$. For this reason, to aid in the development of educational programmes to increase dietary knowledge and intervene in the increasing epidemic of overweight and obesity, we must understand the role of the nutrition transition among college-aged women in Jordan and the impact of Western media and advertising on their dietary values and body image attitudes. A model of nutrition transition which follows economic and demographic transitions developed by Hawks et al. ${ }^{(15)}$ describes a shift from 'physical' eating to 'external' and 'emotional' eating (eating in the absence of hunger) occurring simultaneously with changes in diet composition and eating styles. The model suggests that Western media influences the nutrition transition in part by altering perceptions of ideal body size and shape, leading to restrictive dieting and consequently emotional eating to compensate for food deprivation. Using Hawks et al.'s model ${ }^{(15)}$ as a framework, the purpose of the present study was to evaluate the nutrition transition among college females in Jordan.

\section{Materials and methods}

\section{Sample}

The sample for the current cross-sectional study was taken from undergraduate students attending both public and private universities in Jordan. Since identifying a database of college-aged females was difficult, we partnered with the 'We are all Jordan' youth commission, a government campaign aimed at increasing youth participation in agenda setting. The campaign leadership reviewed the survey instruments. A female research assistant was sent to the meetings and all female attendees were asked to participate in the data collection. There were 255 surveys completed and returned, representing $90 \%$ of the distributed surveys. An informed consent form was provided to the participants explaining the purpose of the study, the voluntary nature of participation and that personal identifying information was not requested. Data were collected using paper-and-pencil surveys, entered manually into a spreadsheet and rechecked for accuracy.

\section{Instruments}

As indicated in previous work, no single scale exists to measure Hawks et al.'s nutrition transition model ${ }^{(10)}$. Thus, the present survey contained a variety of validated instruments, including the Motivation for Eating Scale (MFES), the Restraint Scale (RS), the Eating Attitudes Test (EAT-26), the Sociocultural Attitudes Towards Appearance Questionnaire (SATAQ-3) and the Body Esteem Scale (BES). MFES measures the individual's motivations for eating including physical, external and emotional eating, while the RS measures dietary restraint. EAT-26 was used to measure disordered eating attitudes and behaviours and SATAQ-3 was used to assess the impact of societal and media influences on body image and eating disturbances. Finally, BES assesses general feelings about appearance, weight satisfaction and evaluations attributed to others about one's body and appearance. These instruments have been previously validated among Jordanian women ${ }^{(10)}$. Furthermore, selected demographic variables were included in the questionnaire, as well as Stunkard et al.'s body silhouettes ${ }^{(16)}$. Further descriptions of these instruments have been given elsewhere ${ }^{(10)}$.

Body size preferences were measured using Stunkard's silhouettes ${ }^{(16)}$. Participants were asked three questions: (i) 'Which figure represents your current size?'; (ii) 'Which figure represents your ideal size?'; and (iii) 'Which figure best represents a healthy figure?' Desired change was calculated based on a difference between current size and ideal size. Respondents were then categorized into three groups: those who desired to lose weight, those who desired to gain weight and those who desired no change in their weight.

Another variable of interest was BMI. Respondents were asked to indicate their height and weight, which were used to calculate BMI. Based on the WHO guidelines four different categories of BMI were used: underweight (BMI $<$ $18.5 \mathrm{~kg} / \mathrm{m}^{2}$ ), normal $\left(B M I=18.5-24.9 \mathrm{~kg} / \mathrm{m}^{2}\right)$, overweight $\left(B M I=25 \cdot 0-29 \cdot 9 \mathrm{~kg} / \mathrm{m}^{2}\right)$ and obese $\left(B M I \geq 30 \cdot 0 \mathrm{~kg} / \mathrm{m}^{2}\right)$. Due to low numbers in the overweight and obese categories, these two groups were subsequently merged into one group labelled 'overweight or obese'.

\section{Data analyses}

Data were analysed using the SPSS statistical software package for personal computers release $16 \cdot 0$ (SPSS Inc., Chicago, IL, USA). Conventional frequency tables, $t$ tests and ANOVA were employed to summarize and describe the data. Linear and logistic regression analyses were 
performed controlling for demographic variables to understand the impact of selected variables on eating styles and body size preferences. The level of significance was set at $0 \cdot 05$.

\section{Results}

\section{Demographics}

Table 1 presents the demographic distribution of the sample. The female college students were on average 21 (SD 3) years of age. The majority were single (94.7\%), from medium- to high-income families $(67 \cdot 1 \%)$, in their senior year $(43 \cdot 4 \%)$ and from urban settings ( $87 \cdot 8 \%)$. In addition, most female college students fell in the normal BMI category $(70 \cdot 6 \%)$ with low levels of overweight $(7 \cdot 7 \%)$ and obesity $(5 \cdot 6 \%)$ and a relatively high rate of underweight $(16 \cdot 1 \%)$.

Table 2 presents the mean score and standard deviation for the various scales and subscales, while Table 3 presents the distribution of respondents according to their level of restrained eating, disordered eating attitudes and behaviours, and desired change in body size. Contradictory to our expectations, female college students were more likely to eat based on physical and external hunger cues than based on emotional eating. However, the highest score was on external eating, reflecting a higher rate of social and environmental eating. In addition, they had an alarming rate of restrained eating with $99 \cdot 4 \%$ of the sample scoring higher than the cut-off point of 10 for restrained eating. In addition, disordered eating attitudes and behaviours as measured by EAT-26 indicated that

Table 1 Distribution of participants according to selected demographic variables: female college-aged students, Jordan

\begin{tabular}{lc}
\hline Variable & Percentage $(n$ 255) \\
\hline Age (mean =21, SD = 3 years) & \\
$18-20$ & $40 \cdot 6$ \\
$21-23$ & $50 \cdot 7$ \\
$24+$ & $8 \cdot 7$ \\
Marital status & \\
Single* & $94 \cdot 7$ \\
Married & $5 \cdot 3$ \\
Annual income (Jordanian Dinars) & \\
Low income (0-499) & $34 \cdot 9$ \\
Medium income $(500-999)$ & $40 \cdot 4$ \\
High income $(1000+)$ & $24 \cdot 7$ \\
Year in school & \\
Freshman & $6 \cdot 6$ \\
Sophomore & $23 \cdot 8$ \\
Junior & $26 \cdot 2$ \\
Senior & $43 \cdot 4$ \\
Area of residence & \\
Urban & $87 \cdot 8$ \\
Rural & $12 \cdot 2$ \\
BMl (mean $\left.=22 \cdot 3, \mathrm{SD}=5 \cdot 9 \mathrm{~kg} / \mathrm{m}^{2}\right)$ & \\
Underweight & $16 \cdot 1$ \\
Normal weight & $70 \cdot 6$ \\
Overweight & $7 \cdot 7$ \\
Obese & $5 \cdot 6$ \\
\hline
\end{tabular}

*Includes one divorced female. approximately half of these female college students should be screened for eating disorders since they had scored higher than the cut-off point of 20 .

Furthermore, the mean scores for the BES subscales indicated that these women did not have substantial body esteem issues. For the three subscales, the mean scores were closer to 2 (disagree) and ranged from $2 \cdot 21$ to $2 \cdot 34$. The mean scores for all of the SATAQ-3 subscales fell between 2 (disagree) and 3 (agree), and ranged from $2 \cdot 49$ and $2 \cdot 92$. While $48 \cdot 2 \%$ of the female college students desired to lose weight and $14.4 \%$ desired weight gain indicating a certain level of body dissatisfaction, the mean desired body silhouette change was 0.49 (losing less than one body silhouette).

To better understand the role of Western advertising and media on the nutrition transition stage, we performed

Table 2 Mean scores on the different scales and subscales: female college-aged students, Jordan

\begin{tabular}{lcr}
\hline Scale/subscale & Mean & \multicolumn{1}{c}{ SD } \\
\hline MFES & & \\
$\quad$ Physical & $2 \cdot 8800^{*}$ & $0 \cdot 5752$ \\
External & $2 \cdot 9058^{*} \dagger$ & $0 \cdot 8070$ \\
$\quad$ Emotional & $2 \cdot 3390$ & $0 \cdot 7577$ \\
EAT-26 & $20 \cdot 8300$ & $15 \cdot 3660$ \\
RS & $24 \cdot 2800$ & $2 \cdot 2480$ \\
SATAQ-3 & & \\
$\quad$ Information & $2 \cdot 4860$ & $1 \cdot 1229$ \\
$\quad$ Pressures & $2 \cdot 9202$ & $1 \cdot 2365$ \\
$\quad$ Internalization - general & $2 \cdot 8950$ & $1 \cdot 2693$ \\
$\quad$ Internalization - athlete & $2 \cdot 6720$ & $1 \cdot 2052$ \\
BES & & \\
$\quad$ Body esteem - appearance & $2 \cdot 2500$ & 0.9676 \\
Body esteem - weight & $2 \cdot 3400$ & $1 \cdot 0400$ \\
$\quad$ Body esteem - attribution & $2 \cdot 2110$ & $0 \cdot 9889$ \\
BMl & $22 \cdot 3125$ & $5 \cdot 8762$ \\
Desired change & $0 \cdot 4892$ & $1 \cdot 0992$ \\
\hline
\end{tabular}

MFES, Motivation for Eating Scale; EAT-26, Eating Attitudes Test; RS, Restraint Scale; SATAQ-3, Sociocultural Attitudes Towards Appearance Questionnaire; BES, Body Esteem Scale.

${ }^{*}$ Mean value was significantly different from that of emotional eating $(P<0.05)$. tMean value was significantly different from that of physical eating $(P<0 \cdot 05)$.

Table 3 Distribution of respondents according to restrained eating, disordered eating attitudes and behaviours, and desired change in body size: female college-aged students, Jordan

\begin{tabular}{|c|c|}
\hline Variable & Percentage \\
\hline \multicolumn{2}{|l|}{ RS } \\
\hline Restrained eating (score $10+$ ) & $99 \cdot 4$ \\
\hline No restrained eating (score $<10$ ) & $0 \cdot 6$ \\
\hline \multicolumn{2}{|l|}{ EAT-26 } \\
\hline $\begin{array}{l}\text { Disordered eating attitudes and behaviours } \\
(\text { score } 20+)\end{array}$ & $45 \cdot 2$ \\
\hline $\begin{array}{l}\text { No disordered eating attitudes and behaviours } \\
(\text { score }<20)\end{array}$ & $54 \cdot 8$ \\
\hline \multicolumn{2}{|l|}{ Desired change in body size } \\
\hline $\begin{array}{l}\text { Weight loss (difference between current and ideal } \\
\text { silhouette }>0 \text { ) }\end{array}$ & $48 \cdot 2$ \\
\hline $\begin{array}{l}\text { No change (no difference between current and ideal } \\
\text { silhouette) }\end{array}$ & $37 \cdot 4$ \\
\hline $\begin{array}{l}\text { Weight gain (difference between current and ideal } \\
\text { silhouette }<0 \text { ) }\end{array}$ & $14 \cdot 4$ \\
\hline
\end{tabular}


Table 4 Unstandardized coefficients in linear regression models for independent variables and external eating, restrained eating and EAT26 total score, and odds ratio in logistic regression models for independent variables and desired change in body size: female college-aged students, Jordan

\begin{tabular}{|c|c|c|c|c|c|c|c|c|}
\hline \multirow[b]{2}{*}{ Variable } & \multicolumn{2}{|c|}{ External eating } & \multicolumn{2}{|c|}{ Restrained eating } & \multicolumn{2}{|c|}{ EAT-26 } & \multicolumn{2}{|c|}{ Desired change } \\
\hline & $B$ & $P$ & $B$ & $P$ & $B$ & $P$ & OR & $P$ \\
\hline \multicolumn{9}{|l|}{ Area of residence } \\
\hline Urban & & & $2 \cdot 027$ & 0.016 & & & & \\
\hline Rural & & & & & & & & \\
\hline \multicolumn{9}{|l|}{ SATAQ-3 } \\
\hline Information & & & & & $2 \cdot 846$ & 0.001 & & \\
\hline Pressures & & & $2 \cdot 533$ & 0.012 & & & $6 \cdot 687$ & 0.038 \\
\hline Internalization - general & & & $2 \cdot 504$ & 0.014 & $1 \cdot 941$ & 0.046 & & \\
\hline Internalization - athlete & & & & & & & & \\
\hline \multicolumn{9}{|l|}{ BMI } \\
\hline \multirow{2}{*}{\multicolumn{9}{|c|}{$\begin{array}{l}\text { Underweight } \\
\text { Normal weight }\end{array}$}} \\
\hline & & & & & & & & \\
\hline Overweight or obese & & & $2 \cdot 027$ & 0.035 & $1 \cdot 227$ & 0.020 & $4 \cdot 188$ & 0.003 \\
\hline \multicolumn{9}{|l|}{ Marital status } \\
\hline Married & & & & & & & & \\
\hline Single & $1 \cdot 871$ & 0.036 & & & & & & \\
\hline \multicolumn{9}{|l|}{ Annual income } \\
\hline \multicolumn{9}{|l|}{ Low income } \\
\hline Medium income & & & $1 \cdot 797$ & 0.047 & $2 \cdot 377$ & 0.025 & & \\
\hline High income & $1 \cdot 924$ & 0.025 & $2 \cdot 607$ & 0.001 & $3 \cdot 206$ & 0.003 & & \\
\hline \multicolumn{9}{|l|}{ Year in school } \\
\hline \multicolumn{9}{|l|}{$\begin{array}{l}\text { Freshman } \\
\text { Sophomore }\end{array}$} \\
\hline $\begin{array}{l}\text { Sophomore } \\
\text { Junior }\end{array}$ & 2.991 & 0.003 & & & & & & \\
\hline Senior & $2 \cdot 376$ & 0.019 & & & & & & \\
\hline Constant & $8 \cdot 324$ & $<0.001$ & $14 \cdot 543$ & $<0.001$ & 21.934 & $<0.001$ & & \\
\hline$R^{2}$ adjusted/-2LL & & & & & & & & \\
\hline $\mathrm{N} / \mathrm{df}$ & & & & & & & & \\
\hline
\end{tabular}

EAT-26, Eating Attitudes Test; SATAQ-3, Sociocultural Attitudes Towards Appearance Questionnaire.

the regression of three main independent variables - area of residence, BMI and Western advertising and media (SATAQ-3 scores) $-v$. four dependent variables: external eating, restrained eating, EAT-26 scores and desired change. Linear regression models were employed on the dependent variables external eating, restrained eating and EAT-26 scores. Logistic regression was used for desired change. We also controlled for demographic variables. These included age, marital status, income and education level. Age was insignificant in all of the models and was thus dropped from the final models. Results are presented in Table 4 .

\section{External eating model}

The first model, external eating, revealed that none of the three main independent variables regressed significantly affected the external eating score. However, three demographic variables were associated with the external eating score: (i) single women scored 1.871 points $(P=0.036)$ more on the external eating subscale than married women; (ii) women in the high income bracket scored 1.924 points $(P=0.025)$ higher on the external eating subscale compared with women in the low income bracket; and (iii) junior and senior women scored $2 \cdot 991$ $(P=0.003)$ and $2.376(P=0 \cdot 019)$ points more, respectively, than freshmen women.

\section{Restrained eating model}

In this model, all three main independent variables regressed on the restrained eating scale score were significant. First, the effect of living in urban $v$. rural settings was significant only in this model and indicated that women living in urban areas scored $2 \cdot 027$ points $(P=0 \cdot 016)$ more on the restrained eating scale compared with those women living in rural areas. Second, for every point scored on pressures from the media and internalizing general images in the media, there was an increase of $2.533(P=0.012)$ and 2.504 $(P=0 \cdot 014)$ points, respectively, on the restrained eating scale, indicating the women were more likely to refrain from eating or restrict their energy intake. Being overweight or obese compared with normal weight resulted in a restrained eating score that was $2 \cdot 027$ points higher $(P=0 \cdot 035)$. Increases in income also led to increases in the restrained eating score. Those with medium and high incomes scored $1.797(P=0.047)$ and $2.607(P=0.001)$ points more, respectively, than those with low income.

\section{EAT-26 model}

In this model, two of the three main independent variables regressed were significantly associated. For every point scored on media information regarding body image and internalizing the general images in the media, college-aged Jordanian women scored $2 \cdot 846(P=0 \cdot 001)$ and 1.941 
$(P=0 \cdot 046)$ points higher, respectively, on the EAT-26 scale, indicating elevated disordered eating attitudes and behaviours. Being overweight or obese, compared with normal BMI, resulted in an increase of 1.227 points $(P=0.020)$ on the EAT-26 scale. Similar to the two previous models, increases in income level resulted in higher scores in the EAT-26 model. Those with medium and high incomes scored $2 \cdot 377(P=0 \cdot 025)$ and $3 \cdot 206(P=0 \cdot 003)$ points more, respectively, higher than those with low income.

\section{Desired change model}

The desired change model indicated that for every point scored on pressures from the media, there was a $6 \cdot 7$-fold $(P=0 \cdot 038)$ increased likelihood that college-aged Jordanian women wanted to lose weight. Those who were overweight or obese, as compared with those of normal weight, were also $4 \cdot 2$ times $(P=0 \cdot 003)$ more likely to want to lose weight. All models resulted in directional consistency with expected results based on previous data.

\section{Discussion}

As previous studies have shown, Jordan is rapidly progressing through the stages of the nutrition transition ${ }^{(11,12)}$. Therefore, it is not unexpected that this movement away from traditional values is affecting body image attitudes and dietary behaviours in college-aged women in Jordan. The BMI distribution of college women in this sample may be linked to their eating behaviours. Our findings revealed that the study population of college-aged women showed high levels of restrained eating and disturbing rates of disordered eating attitudes and behaviours even above those of Jordanian adolescents ${ }^{(17)}$. This may explain the relatively high rates of underweight in the sample. However, these college-aged women were more likely to eat based on external and physical hunger cues than emotional hunger cues, contrary to what was previously observed in the general population of Jordanian women ${ }^{(10)}$, suggesting that college-aged women may be less advanced in their development through the nutrition transition. This can also be seen by their relatively low rates of overweight and obesity.

That college-aged women in the high- and mediumincome brackets are more prone to external eating, restrained and disordered eating reflects correctly the nutrition transition which proposes that those more affluent in society are affected first. College-aged women who were not married and those college-aged women who were more progressed in their education were also more prone to external eating. One reason for this might be that college females who are juniors and seniors and those who are single may have developed stronger social networks that lead to higher rates of external eating. Also increases in stress levels may explain a portion of the rise in restrained and disordered eating ${ }^{(18)}$. Since external eating among college women has been associated elsewhere with a higher intake of fatty foods than restrictive or emotional eating ${ }^{(19)}$, a propensity towards external eating as one progresses through college provides rationale for an intervention in the freshman year of college.

As seen in the general population of Jordanian women, college-aged women retained normal levels of body esteem and low levels of body dissatisfaction, signifying that the women may preserve traditional attitudes that favour a 'normal' body size ${ }^{(10)}$. Additionally, those women who did desire changes in body size maintained healthy and reasonable attitudes regarding weight loss and weight gain, preferring changes that measured less than one body silhouette and stayed closer to the previously mentioned 'normal' body size. Overall, collegeaged women sustained body esteem levels that were normal despite the high rates of restrained eating and disordered eating attitudes and behaviours that indicate the possible development of future eating disorders.

Although in general Jordanian women did not believe that exposure to Western media directly influences their body esteem and dietary attitudes, we can consider that Western concepts and ideals that promote thinness may be internalized and these women may not recognize how they are being persuaded by media messages ${ }^{(10)}$. Collegeaged women may be specifically susceptible as evidenced by comparing these results with the general population of Jordanian women. The pressures felt to have a certain body type from Western media as measured by mean scores from the SATAQ-3 instrument were 2.92 in the present study of college-aged women as opposed to $2 \cdot 11$ in a previous study from Jordanian women of all ages ${ }^{(10)}$. The shift in direction in mean scores for the SATAQ-3 instrument suggests that Western media is beginning to be recognized among the younger generation as having some effect and is consistent with trends in Jordanian adolescents $^{(20)}$. It seems for this population that Western advertising and media explains, in part, the nutrition transition as it relates to eating style (restrained eating but not external eating) and body size preferences among Jordanian college-aged females.

Furthermore, college-aged women may be experiencing the same cultural conflict as seen in the general population of Jordanian women; they are torn between traditional values that dictate cultural norms regarding body size and beauty and a Westernized society that promotes thinness and increased access to pre-packaged and larger portions of food ${ }^{(10)}$. College-aged women may be simply unaware of their own struggle and why they may be partaking in certain dieting behaviours such as the high rates of restrained eating found in the present study.

\section{Limitations}

Although the cross-sectional study design introduces the possibility of selection bias, randomized sampling of students from multiple institutions across Jordan presents 
challenges as no database exists from which to sample. The sample included participants from both public and private institutions across Jordan, increasing the representation of varying types of college-aged women. Consequently, the sample matched well with the general population demographically but had an over-representation of students with middle-to-high income which may be due to the incorporation of private universities into the sampling frame. Private universities in Jordan are expensive and are often exclusive to middle- to high-income families who are able to afford it.

\section{Implications and future research}

Our findings reveal that, without proper intervention, college-aged women's rates of overweight and obesity have the potential to increase as they enter adulthood given their reported attitudes and behaviours. Therefore, it is necessary to develop public health interventions for this age group to promote appropriate dieting behaviours and help to maintain healthy body esteem. Additionally, there is a need to increase awareness of the negative psychological and physical consequences of restrained dieting and disordered eating attitudes and behaviours, emphasizing that such behaviour may lead to greater weight fluctuations, reduced body esteem and the development of more serious eating disorders ${ }^{(21,22)}$. Education regarding media images of ideal body size and its impact on body satisfaction may also be warranted.

Future research needs to measure the current levels of eating disorders within the population as well as develop measures to understand the impact of Western influences on body image and daily food intake. Additionally, similar research needs to be extended to include males and adolescents who may also be experiencing changes in dietary behaviour and body image. Of particular importance are adolescents who, compared with their parents, will have greater and longer exposure to Western media over their lifetime and will be further removed from traditional values that include dietary behaviour and attitudes as the nutrition transition progresses in Jordan. This information combined with our results may form the basis of future nutrition education interventions that aim at improving dietary behaviours and perceptions of ideal body size while curbing overweight and obesity rates.

\section{Acknowledgements}

This research did not receive any specific grant from any funding agency in the public, commercial or not-forprofit sector and there are no conflicts of interest to disclose. H.N.M. developed the research question, collected, entered and analysed the data, and contributed to writing the manuscript. R.L. and T.C. contributed to the writing of the manuscript. R.L. also discussed and commented on the results and their implications.

\section{References}

1. Drewnowski A (2000) Nutrition transition and global dietary trends. Nutrition 16, 486-487.

2. Popkin BM (2001) Nutrition in transition: the changing global nutrition challenge. Asia Pac J Clin Nutr 10, Suppl., S13-S18.

3. Popkin BM (2009) Global changes in diet and activity patterns as drivers of the nutrition transition. Nestle Nutr Workshop Ser Pediatr Program 63, 1-10.

4. Popkin BM (2002) An overview on the nutrition transition and its health implications: the Bellagio meeting. Public Health Nutr 5, 93-103.

5. Popkin BM (2006) Global nutrition dynamics: the world is shifting rapidly toward a diet linked with noncommunicable diseases. Am J Clin Nutr 84, 289-298.

6. Popkin BM \& Gordon-Larsen P (2004) The nutrition transition: worldwide obesity dynamics and their determinants. Int J Obes Relat Metab Disord 28, Suppl. 3, S2-S9.

7. Maire B, Lioret S, Gartner A et al. (2002) Nutritional transition and non-communicable diet-related chronic diseases in developing countries. Sante 12, 45-55.

8. Baillie K (2008) Health implications of transition from a planned to a free-market economy - an overview. Obes Rev 9, Suppl. 1, 146-150.

9. Craven KL \& Hawks SR (2006) Cultural and western influences on the nutrition transition in Thailand. Promot Educ 13, 14-20.

10. Madanat HN, Brown RB \& Hawks SR (2007) The impact of body mass index and Western advertising and media on eating style, body image and nutrition transition among Jordanian women. Public Health Nutr 10, 1039-1046.

11. Madanat HN, Troutman KP \& Al-Madi B (2008) The nutrition transition in Jordan: the political, economic and food consumption contexts. Promot Educ 15, 6-10.

12. Khader Y, Batieha A, Ajlouni $\mathrm{H}$ et al. (2008) Obesity in Jordan: prevalence, associated factors, comorbidities, and change in prevalence over ten years. Metab Syndr Relat Disord 6, 113-120.

13. Tsai CY, Hoerr SL \& Song WO (1998) Dieting behavior of Asian college women attending a US university. J Am Coll Health 46, 163-168.

14. Yager Z \& O'Dea JA (2008) Prevention programs for body image and eating disorders on university campuses: a review of large, controlled interventions. Health Promot Int 23, 173-189.

15. Hawks SR, Merrill RM, Madanat HN et al. (2004) Intuitive eating and the nutrition transition in Asia. Asia Pac J Clin Nutr 13, 194-203.

16. Stunkard AJ, Sorensen T \& Schulsinger F (1983) Use of the Danish Adoption Register for the study of obesity and thinness. Res Publ Assoc Res Nerv Ment Dis 60, 115-120.

17. Mousa TY, Al-Domi HA, Mashal RH et al. (2010) Eating disturbances among adolescent schoolgirls in Jordan. Appetite 54, 196-201.

18. Kandiah J, Yake M, Jones J et al. (2006) Stress influences appetite and comfort food preferences in college women. Nutr Res 26, 118-123.

19. Anschutz DJ, Van Strien T, Van De Ven MO et al. (2009) Eating styles and energy intake in young women. Appetite 53, 119-122.

20. Mousa TY, Mashal RH, Al-Domi HA et al. (2010) Body image dissatisfaction among adolescent schoolgirls in Jordan. Body Image 7, 46-50.

21. Laessle RG \& Kikker S (2008) Resting metabolic rate in young women classified as restrained or unrestrained eaters. Physiol Behav 95, 542-543.

22. Anschutz DJ, Van Strien T \& Engels RC (2008) Exposure to slim images in mass media: television commercials as reminders of restriction in restrained eaters. Health Psychol 27, 401-408. 